Relations industrielles

Industrial Relations

\title{
Economic Analysis and Industrial Management, par Jacques Lesourne, Prentice-Hall, Inc., Englewood Cliffs, N.J., 1963, 631 pages.
}

\section{Bertrand Belzile}

Volume 19, numéro 1, janvier 1964

URI : https://id.erudit.org/iderudit/1021386ar

DOI : https://doi.org/10.7202/1021386ar

Aller au sommaire du numéro

Éditeur(s)

Département des relations industrielles de l’Université Laval

ISSN

0034-379X (imprimé)

1703-8138 (numérique)

Découvrir la revue

Citer ce compte rendu

Belzile, B. (1964). Compte rendu de [Economic Analysis and Industrial Management, par Jacques Lesourne, Prentice-Hall, Inc., Englewood Cliffs, N.J., 1963, 631 pages.] Relations industrielles / Industrial Relations, 19(1), 133-134. https://doi.org/10.7202/1021386ar

Tous droits réservés @ C Département des relations industrielles de l’Université Laval, 1964
Ce document est protégé par la loi sur le droit d'auteur. L'utilisation des services d’Érudit (y compris la reproduction) est assujettie à sa politique d'utilisation que vous pouvez consulter en ligne.

https://apropos.erudit.org/fr/usagers/politique-dutilisation/ 
crets utilisables pour fins de discussion et d'onalyse.

Enfin, les principoux textes législotifs pertinents ou sujet sont fournis en oppendice, soit dans leur teneur totale, soit en dé larges extraits.

Somme toute un des manuels les mieux faits et parmi les plus utiles, outant aux proticiens qu'aux étudiants en lo matière.

\section{Jean-Réal Cordin}

Le Statut des Entreprises publiques, par A G. Delion, Collection de l'Administration nouvelle, Berger-Levrault, Paris, 1963, 268 pages.

L'on n'a plus à démontrer, il me semble, l'importance des entreprises publiques dans la plupart des pays y compris ceux de I'Occident. En ce qui a trait à la France, André DELION a déjà publié plusieurs études sur le sujet, notomment, PEtat et les entreprises publiques (Sirey, Poris, 1959).

Dans Le Statut des Entreprises publiques $\gg$, I'auteur examine systématiquement et fort judicieusement les principales interrelations entre d'une part, l'entreprise publique et d'autre part, les organismes de contrôle que sont le porlement, le gouvernement et le Conseil d'Etat. En guise d'introduction, DELION donne quelques notes historiques et indique les règles relatives à la création, d̀ la notionalisotion et d̀ la disparition des entreprises publiques. L'ouvroge est très à point et fort bien rédigé

L'opproche est foncièrement formelle et juridique. Ce qui explique sans doute pourquoi I'on a ignoré le rôle des partis et celui de l'opinion publique Les discussions et les critiques sont rares. Les préoccupations d'ordre administrotif, politique et économique sous-jocentes sont rarement exprimées. II existe à cet égard un contraste frappant entre l'ouvrage de DELION et certaines études des onnées d'après guerre. notamment celles de Ventanat, de Juliot de la Morandière et de Lavergne qui mettaient en cause, sous différents aspects, l'idée même de la nationalisation. Cette idée étont mointenant devenue occeptoble il appartient ou juriste de formuler les nouvelles règles fondamentales d'organisation générale, c'est-à-dire, le stotut.

II va sans dire que I'on peut difficilement comporer le statut des entreprises publiques en Fronce et au Québec. Même si l'on fait obstraction des différences d'ordre quantitatif qui sont considérobles (en 1963, lo France comptait 150 entreprises publiques, le gouvernement canadien une quarontaine et le Québec une dizaine) il n'en reste pos moins que le contexte global est loin d'être identique. Lo planificotion économique est ici embryonnaire, les responsabilités sont portagées entre les collectivités provincioles et centrales, enfin la gestion publique commerciole est encore une source de controverses.

Sous l'angle restreint des rapports entre les entreprises publiques et les autorités de tutelle, il est vrai que nous observons ici oussi le principe de l'autonomie de gestion. Par contre, les moyens de contrôle diffèrent sensiblement. II n'y a pos ou Québec, par exemple, de commissaires de gouvernement, ni Commissions de Vérification des comptes des entreprises publiques, ni bien entendu, de Conseil d'Etot. De plus, les conseils d'administration n'obéissent pos ò lo formule de composition tripartite. Quont d la surveillance parlementaire, la nôtre frise I'insignifiance. L'Assemblée Législative n'a élaboré oucun méconisme spécial de contrôle. Evidemment, l'on peut expliquer ces différences par une foule de raisons dont certaines sont attribuables oux structures même de l'administration générale. Par contre, il fout bien dire qu'd mon sens, I'on a peu ressenti ici la nécessité d'une surveillonce plus étroite tout simplement à cause du petit nombre d'entreprises publıques. Le besoin devrait éventuellement créer l'organe.

\section{André Gélinas}

\section{Economic Analysis and Industrial Manage-} ment, par Jacques Lesourne, Prentice Holl, Inc., Englewood Cliffs, N.J., 1963, 631 pages.

$\mathrm{Ce}$ volume a déjò paru en françois en 1958 sous le titre \& Technique Economique et Gestion Industrielle $>$ II constitue une lecture probablement des plus profitables pour le futur ingénieur-économiste. En effet, nous croyons que ce livre s'odresse surtout aux ingénieurs qui se dirigent vers l'odministration de l'entreprise et qui partont désirent ocquérir une connaissonce de la théorie économique appliquée d̀ la gestion industrielle. Beaucoup d'économistes peuvent égolement tirer profit de lo formulation des principes économiques en termes mothématiques et statistiques. II ne 
faut pas oublier non plus que l'auteur se préoccupe constomment de l'application de la science économique à l'administration de I'entreprise.

Après une excellente préface du professeur Mourice Allais, M. Lesourne traite, assez longuement, dans son introduction, de la méthode scientifique et de la technique économique et puis du choix des critères économiques. Le volume comprend trois grandes parties.

La première réfère d̀ l'économétrie au service de l'entreprise. On y retrouve des chapitres sur les études commerciales, sur I'analyse théorique de la demande et sur les fluctuations de la conjoncture et deux derniers chapitres, I'un portant sur la discussion des données chiffrées et l'autre comprenant des exemples d'application de l'économétrie.

Le deuxième partie traite de la structure des coûts de l'entreprise. Deux courts chapitres: un premier sur la notion de coût: de la comptabilité à l'économique et un deuxième sur l'amortissement. Dans le troisième chapitre, Monsieur Lesourne fait une excellente analyse des coûts marginaux: théorie pure et applications concrètes.

Puis lo dernière partie développe les problèmes de synthèse: la régulation de la production et la gestion des stocks, la détermination des programmes optimums, les problèmes $d$ 'investissement et le calcul du revenu actualisé et enfin la politique de prix. Le seul chapitre réellement difficile est le deuxième de cette partie, chapitre qui présente des modèles mathématiques dont principalement le programme linéaire.

Dans une conclusion non moins excellente que les parties précédentes, l'auteur traite de la technique économique et de I'organisation de l'entreprise. II définit les missions des ingénieurs-économistes et parle du service économique dans l'entreprise.

\section{Bertrand Belzile}

Démocratie aujourd'hui. Ouvrage en collaboration par P. Antoine, F. Bloch-Lainé, F. Bourricaud, G. Burdeau, J.-Y. Calvez, A. Jeannière, G. Lavau, J.-L. Quermonne. Bibliothèque de la recherche sociale, Editions Spes, Paris, 1963, 188 pages.

《Autont la conviction démocratique est répandue aujourd'hui, autant elle manque le plus souvent de solide justification 》.
Cette affirmation que I'on trouve du début de l'introduction de cet ouvrage est pleinement vraie en ce qui concerne notre pays. Aussi nous croyons que les différents collaborateurs appartenant chacun d̀ des disciplines diverses ont rendu un grand service à tous ceux qui sont soucieux du fonctionnement efficace et de la survie de la démocratie.

On reprend ici, pour les rendre accessibles à un public plus vaste, une série d'articles publiés dans la revue de l'Action populaire en 1960-61-62.

L'ouvrage est divisée en deux parties: «Les raisons de la démocratie » et \& La démocratie en pratique ». La première partie comprend trois essais: «Du pouvoir personnel à la démocratie », «Démocratie et participation 》, «Liberté et pouvoir». La seconde en comprend cinq: «Destiin de l'idée démocratique 》, «Démocratiser la république », «La Polyarchie ou la société raisonnable», «Pouvoir économique et démocratique », \&Des démocraties qui fonctionnent $\gg$.

Souf un chapitre où il est particulièrement question de la situation française, tous les outres possèdent un caractère général.

Les auteurs ovaient pour préoccupation de réexaminer les raisons de la démocratie et d'apprécier le fonctionnement actuel des régimes démocratiques. L'ensemble de ces analyses, sans constituer un traité complet sur la question, est sûrement une contribution très valable et susceptible d'éclairer tous ceux qui veulent revivifier nos sociétés qui sont en train de pérécliter sous la menace du «consentement» et du «contentement 》.

\section{Gérard Dion}

\section{LES ETAPES DU SYNDICALISME AGRICOLE} EN FRANCE, Dr Louis Prugnaud, Ed. de I'Epi, Paris, 1963, 283 poges.

A tous ceux épris d'histoire rurale, Les Etapes du Syndicalisme agricole en France retracent comment celui-ci, profondément influencé par les partis politiques, a rendu à l'agriculture française d'importants services économiques. Aussi curieux que celo puisse paraître, il n'existe pas d'étude d'ensemble sur I'histoire syndicale agricole et l'auteur lui-même cite cette phrase extraite de I'ouvrage magistral de Marcel Bloch, Les Caractères originaux de I'histoire rurale 